Ann. Biol. anim. Bioch. Biophys., I966, 6 (x), 73-92.

\title{
L'AMMONIAC DANS LE TUBE DIGESTIF DES RUMINANTS - SA SIGNIFICATION
}

\author{
B. GAUSSERES \\ avec la collaboration technique de Madeleine Vermorat \\ Laboraloire des Métabolismes, \\ Centre national de Recherches zootechniques, 78 - Jouy-en-Josas
}

SOMMAIRE

Cette étude rend compte des résultats de dosages d'ammoniac effectués sur les contenus de rumen d'animaux soumis à différents régimes. Les prélèvements de contenu ont été effectués selon plusieurs techniques. L'une d'entre elles a été mise au point au laboratoire. Ia discussion porte sur la validité et les possibilités d'interprétation des différents résultats.

\section{INTRODUCTION}

Chez les ruminants, les aliments sont soustraits pendant un temps plus ou moins long (de to heures à 5 jours) à la digestion classique par les enzymes de l'animal et soumis à des fermentations dues à des organismes symbiotiques (bactéries et protozoaires). Simultanément à cette action enzymatique et chimique a lieu une action mécanique et physique tout aussi importante. La morphologie et le fonctionnement du rumen dans lequel se situe la plus grande partie de ces phénomènes sont tels qu'il est très difficile d'évaluer de façon quantitative (et même quelquefois qualitative) les transformations subies par les aliments. L'azote alimentaire se trouve pour une part sous forme protéique, et pour une autre part sous forme non protéique. Cette dernière fraction est constituée essentiellement d'acides aminés et amides libres. Quarante à quatre-vingt-dix p. Ioo des protéines et près de Ioo p. Ioo de l'azote non protéique sont métabolisés dans le rumen par la population microbienne (Mc DONALD, I954; Mc Donald et Hil, I957 ; WEI,IER et al., I958 ; GaUs SERES Et FAUCONNEAU, I965). 
L'ammoniac représente un stade intermédiaire ou final de ce métabolisme (final, s'il est absorbé à travers la paroi). Mc DONALD, I952 ; AnNison et al., I954, ont les premiers attiré l'attention sur son accumulation dans les jus de rumen. Les valeurs trouvées par différents auteurs s'échelonnent entre quelques milligrammes d'azote ammoniacal par Ioo $\mathrm{m} 1 \mathrm{de}$ jus (FAUCONNEAU et GAUSSERES, I96I; LEWIS, I962) et I30 mg (Johns, I956). Une partie de cet ammoniac passe dans le sang des veines du rumen (Mc Donalid, I948). On a pu mettre en évidence le parallèlisme entre les taux d'ammoniac dans le rumen d'une part et les taux d'ammoniac et d'urée dans le sang d'autre part (LEWIS, I957 ; HOGAN, I96I ; GAR'TNER, I963 ; GaERTNER, DECKer et Hil, I96I ; SuTTON et al., I963). Comme l'urée est le produit terminal du métabolisme de l'azote, on a admis que cette production d'ammoniac (transformée en urée par le foie) correspondait à une perte d'azote. Quelques études et notamment celles de ChaImers et al. (I954) ont montré que le bilan azoté était d'autant plus faible que la production d'ammoniac au niveau du rumen était plus élevée, pour des rations dont le taux azoté était normal. Cette production serait proportionnelle à la solubilité des protéines (LEWIS, I962), bien que LITTLE et Burroughs ( 1963 ) ne trouvent pas une relation bien nette entre solubilité et production d'ammoniac.

Comme les acides gras volatils qui permettent d'évaluer les fermentations des glucides, l'ammoniac est utilisé comme test du métabolisme des matières azotées d'une ration. Ainsi, depuis I959 nous dosons systématiquement 1'ammoniac dans les contenus de rumen. Pour tenter d'expliquer certains phénomènes observés, nous avons été amené à faire une étude critique de la méthodologie et à reviser certaines des conceptions habituellement trouvées dans la littérature.

\section{MATÉRIEL, FXPÉRIMENTAL ET MÉTHODES}

Nous avons effectué de nombreux dosages sur des animaux d'âges et d'états physiologiques très différents, soumis à divers régimes (les plus couramment utilisés dans la pratique). Ces animaux, dont nous indiquons les caractéristiques dans les tableaux, appartenaient pour la plupart à la Station de Recherche sur l'Élevage du Ruminant et étaient utilisés à d'autres fins expérimentales.

Trois techniques de prélèvements étaient utilisées : prélèvements sur animaux abattus, prélèvements sur animaux fistulisés avec pompages sporadiques, prélèvements sur animaux fistulisés avec pompages automatiques, l'appareillage demeurant in situ dans ce dernier cas. Pour les animaux abattus, après homogénéisation $d u$ contenu, les échantillons moyens étaient pressés à la main, filtrés sur quatre épaisseurs de mousseline, puis centrifugés. Les pompages sporadiques étaient effectués, canule ouverte, à l'aide d'une machine à traire (deux ou trois litres de liquide étaient aspirés au total dans les différentes parties du rumen, puis filtrés et centrifugés).

La technique des prélèvements automatiques a été exposée par ailleurs (GAUSSERES, I965). Une crépine immergée dans le rumen est reliée à une pompe qui fonctionne à intervalles de temps réguliers. Le jus prélevé est récolté dans un collecteur de fractions maintenu à ooC. La crépine peut demeurer en place plusieurs semaines sans inconvénient.

Les premiers échantillons (I959 et I960) ont été analysés immédiatement après le prélèvement. Par la suite, nous avons conservé les jus, en abaissant leur pH entre 4 et 5 avec de l'acide sulfurique et en les congelant à - I $5^{\circ} \mathrm{C}$. Dans le cas des prélèvements classiques, les jus extraits ont été obtenus par centrifugation du liquide provenant de la filtration sur mousseline du contenu brut. Dans le cas des prélèvements automatiques, le liquide pompé est filtré à travers une épaisseur constante de mousse de matière plastique (polyuréthane); sa teneur en matières sèches est faible et peu variable (pour un régime donné) ce qui permet de ne pas centrifuger. 
Les dosages ont tous été effectués par distillation à l'appareil de Parnas-Wagner. Les jus, centrifugés (ou non) étaient amenés à pH 8,5-9 avec une solution de borate de soude et l'ammoniac, recueilli dans une solution d'acide borique, dosé avec de l'acide chlorhydrique 0,or4 $\mathrm{N}$.

\section{Enregistrement du comportement alimentaire}

Parallèlement aux prélèvements automatiques de jus de rumen (et dans ce cas seulement), nous enregistrons le comportement alimentaire des animaux. La technique est inspirée de celle utilisée par RuckBUSH (I963). Nous avons seulement transformé le dispositif d'enregistrement. Une poire élastique est fixée par des sangles sous la mâchoire de l'animal et est reliée à un manomètre par un tube de caoutchouc. L'axe du manomètre est solidaire d'un potentiomètre électrique dont les variations de tension sont enregistrées par un enregistreur galvanométrique sur papier métallisé gradué. La vitesse de déroulement du papier est de $\mathrm{r} \mathrm{cm}$ par 5 minutes (lecture facile et rapide des résultats). L'autonomie de fonctionnement est d'environ six jours. La durée de consommation enregistrée n'est malheureusement pas exactement proportionnelle à la quantité consommée. Les aliments sont donc pesés trois fois par jour. L'intérêt du comportement alimentaire est qu'il donne le moment où ont lieu les repas, leur durée, leur nombre et la vitesse d'ingestion, connaissant avec exactitude la quantité consommée entre les heures de pesées.

\section{RÉSULTATS}

Les différents tableaux et graphiques réunissent les résultats de dosages effectués depuis I959 sur de nombreux animaux soumis à différents régimes, les méthodes de prélèvement étant très variées.

\section{ÉTUDE DE RÉGIMES}

\section{Expérience $I$ : Etude sur animaux abattus}

Le tableau I donne les résultats relatifs aux contenus provenant d'animaux (I48 au total) abattus à des temps précis après un repas. Pour l'ensemble des régimes (I r régimes étudiés), les taux d'ammoniac sont rarement élevés et ne dépassent pas 46,7 $\mathrm{mg} \mathrm{d'N} \mathrm{NH}_{3} /$ IOO $\mathrm{ml}$ de jus (régime composé de foin et d'aliment concentré). Même les fourrages verts tels que le ray-grass et le chou n'entraînent pas de taux supérieurs à $30 \mathrm{mg}$. Il y a une grande variabilité des résultats pour des animaux de même état physiologique soumis au même régime. C'est entre $3 \mathrm{~h}$ et $5 \mathrm{~h}$ après le repas que la variabilité est la plus faible, notamment pour les vaches et les taurillons recevant du foin de luzerne. Cela tient sans doute à ce que les animaux ont des niveaux de consommation très voisins. Au-delà de 5 h, par contre, plus le temps écoulé depuis le dernier repas est élevé, plus semble-t-il la variabilité est grande.

Nous avons essayé, pour quelques régimes, de calculer les quantités totales d'ammoniac présentes dans le rumen (tab1. 2). Nous avons pour ceci tenu compte du poids du contenu et de sa teneur en eau. L'éventail des valeurs ammoniac total est semble-t-il moins ouvert que celui des valeurs donnant la concentration. En effet, $94 \mathrm{p}$. Ioo des valeurs (indépendamment de l'heure des prélèvements) donnant la concentration se situent entre 0,7 et $25\left(\mathrm{mg}\right.$ de $\mathrm{N} \mathrm{NH}_{3} /$ Ioo $\mathrm{ml}$ ) alors que pour les quantités totales, $92 \mathrm{p}$. Ioo des valeurs sont comprises entre I et Io (en $\mathrm{g}$ deNNH${ }_{3}$ ). 


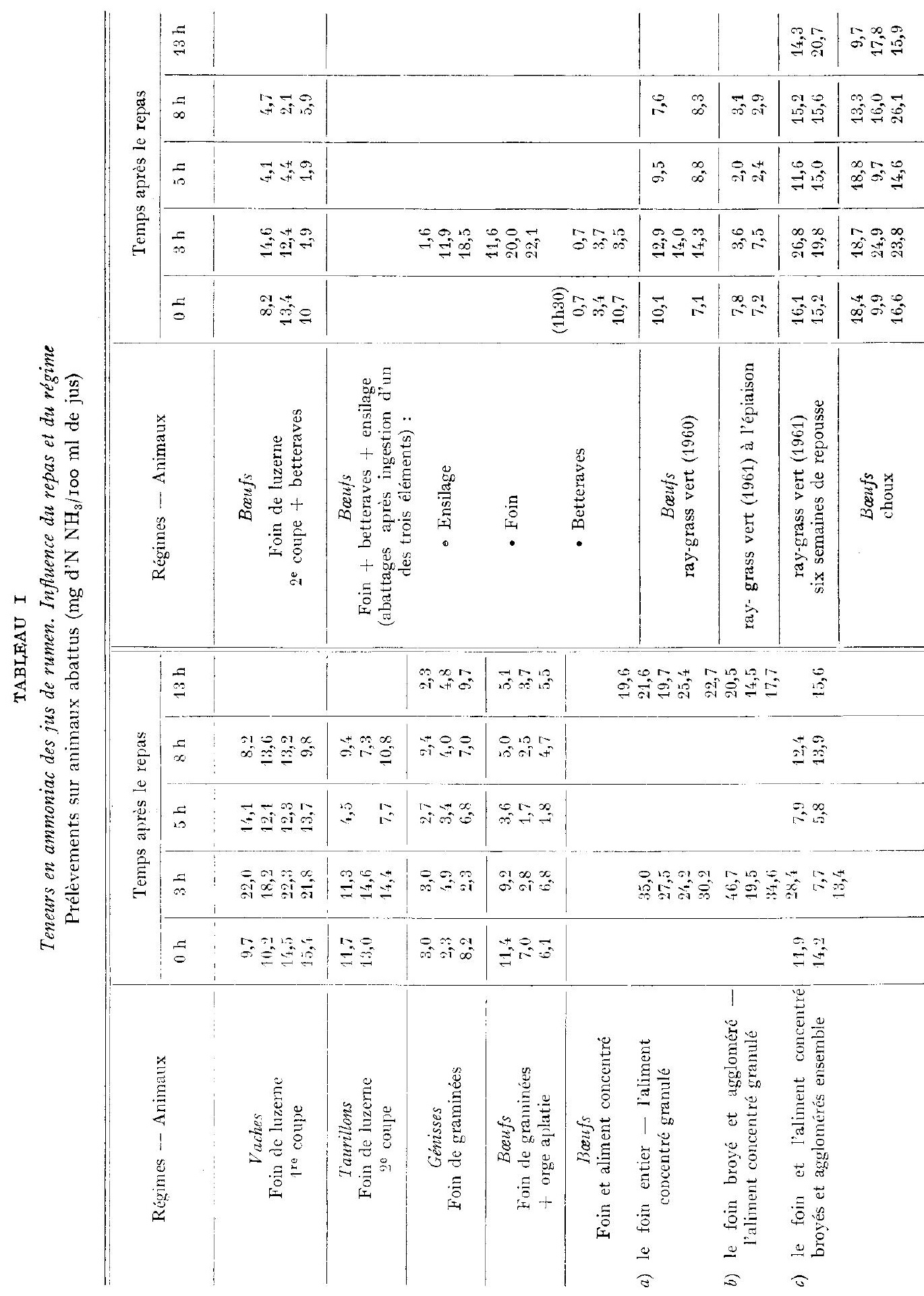




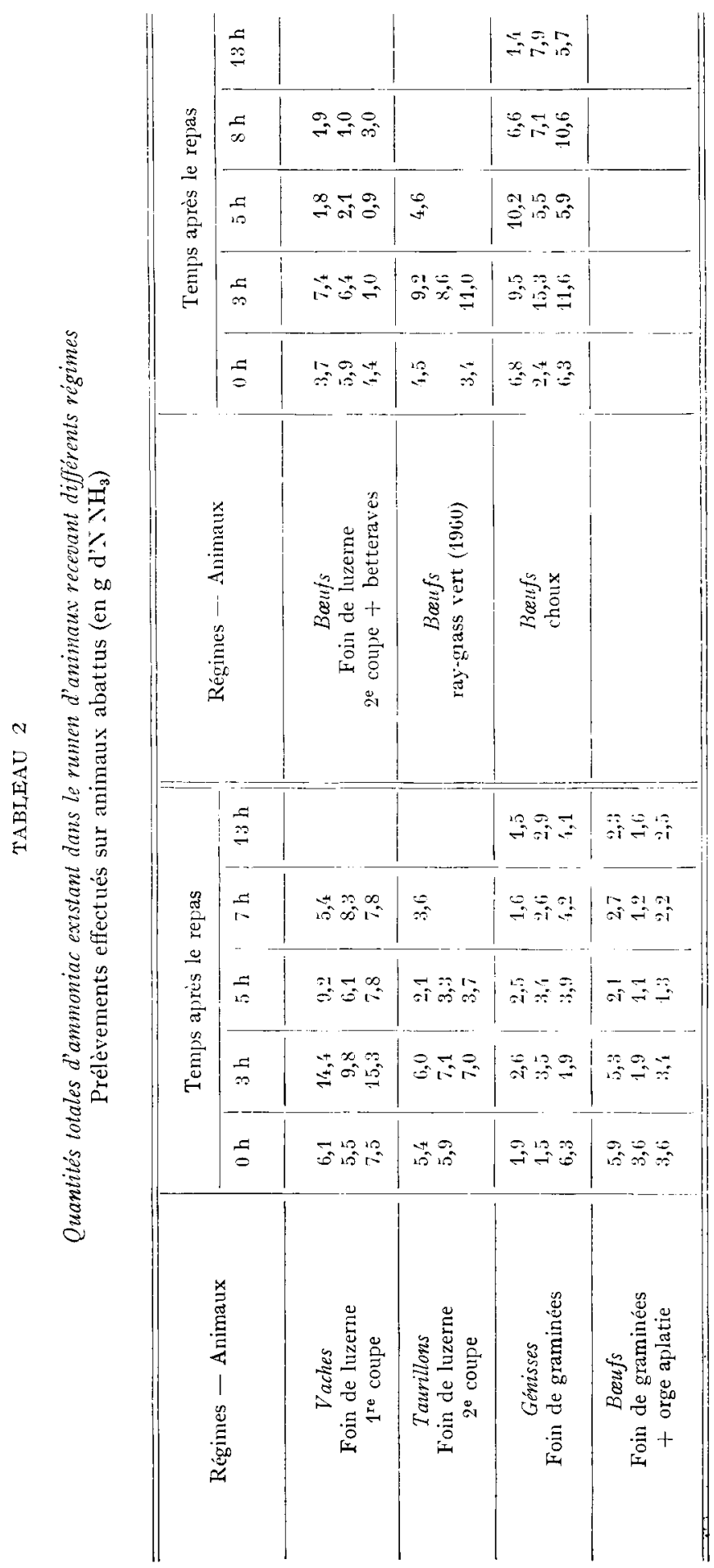


B. GAUSSERES

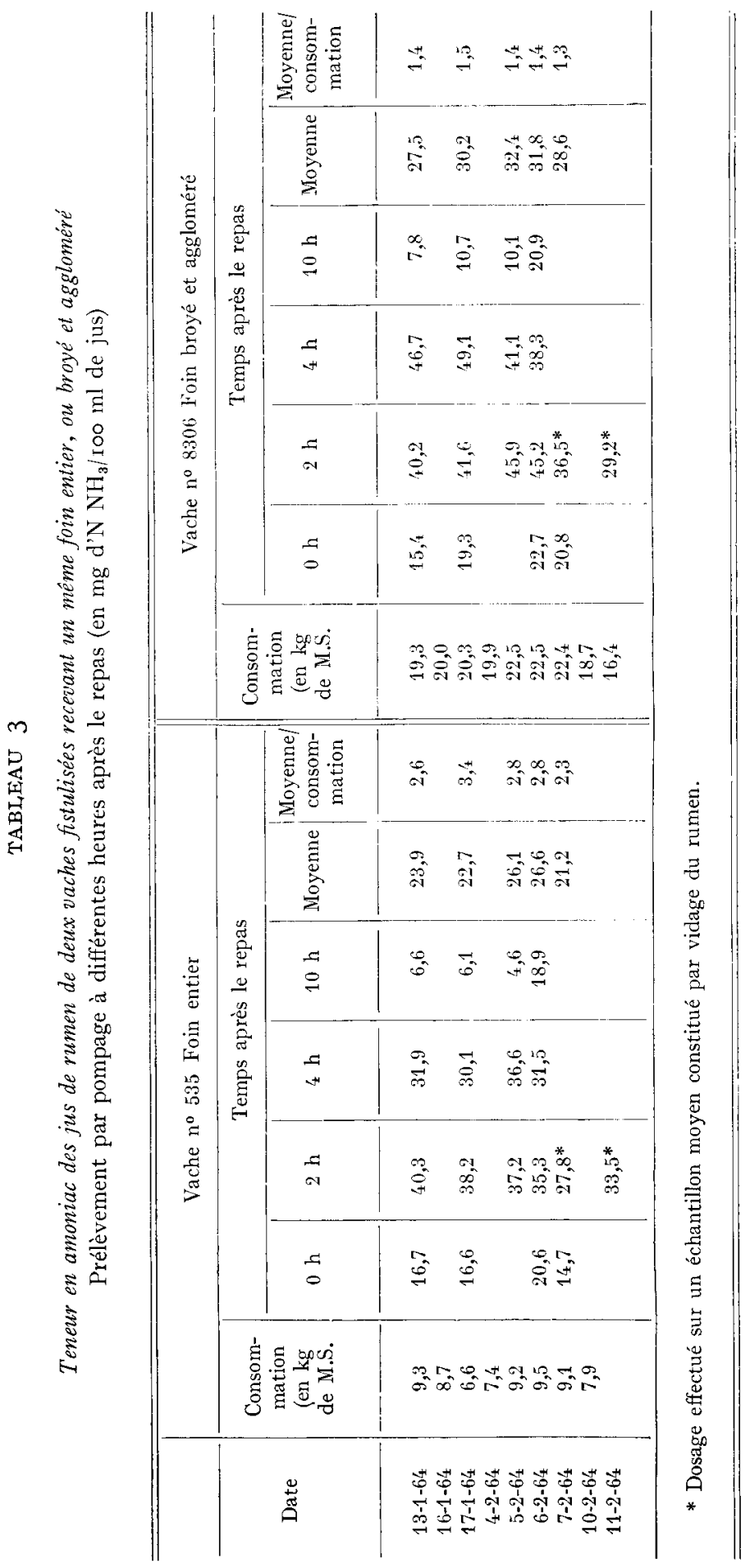




\section{Expérience $I I$ : Étude sur animaux fistulisés}

I $^{0}$ Forme physique du foin.

Dans le tableau 3, nous donnons les résultats d'une comparaison, sur deux vaches fistulées, de deux modes de présentation d'un même foin distribué entier ou broyé et aggloméré. Les concentrations d'ammoniac augmentent dans le même sens que les quantités consommées, mais dans des proportions beaucoup plus faibles que celles-ci. Ainsi, en moyenne, on obtient $21-27 \mathrm{mg} / \mathrm{I} 00 \mathrm{ml}$ pour le foin entier et $27-32$ pour le foin broyé (les consommations moyennes journalières étant respectivement $9 \mathrm{~kg}$. et $20 \mathrm{~kg}$. de M.S.). Si l'on rapporte la concentration moyenne journalière de l'ammoniac à la quantité consommée (nous ne connaissons malheureusement pas les quantités totales d'ammoniac), on trouve des valeurs comprises entre 2,3 et 3,4 pour le foin entier et entre I, 3 et $I, 5$ pour le foin broyé. Ce rapport est donc, pour le foin broyé, plus faible et plus constant. Pour une même quantité de matières azotées ingérée, il doit donc se former moins d'ammoniac. Le foin utilisé pour cette expérience contenait $\mathrm{I}_{4} \mathrm{p}$. Ioo de matières azotées totales (dans la matière sèche), $6,4 \mathrm{p}$. Ioo étant sous forme non protéique (soit $46 \mathrm{p}$. Ioo de l'azote total composésessentiellement d'acides aminés libres, amides et peptides).

\section{$2^{\circ}$ Mise à l'herbe.}

En I963, nous avons suivi l'évolution, au moment de la mise à l'herbe (luzerne), des taux d'ammoniac des contenus de deux vaches fistulisées (graph. r). Avant la mise à l'herbe (régime de foin, ensilage, betteraves, aliment concentré), les quantités d'azote ammoniacal sont comprises entre $\mathrm{I}-5 \mathrm{mg}$ et $20 \mathrm{mg}$ par $100 \mathrm{ml}$. Au cours de la journée, il semble y avoir deux maxima, l'un vers Io h-II $h$, l'autre vers I $7 \mathrm{~h}$-I $8 \mathrm{~h}$. Les quantités consommées des différents aliments et les heures des distributions sont reportées sur le graphique. Au moment du passage au régime d'herbe, les taux d'ammoniac augmentent atteignant 45 pour l'animal qui consomme le plus ( $48 \mathrm{~h}$ après que la tation se compose d'herbe uniquement). Le maximum est atteint approximativement à $\mathrm{I} 2 \mathrm{~h}$. Comme les dosages n'ont pas été faits pendant la nuit, il est difficile d'interpréter avec précision les courbes d'évolution. Quinze jours après la mise à l'herbe, les taux d'ammoniac reviennent sensiblement au niveau du régime hivernal sans que la consommation de matière sèche ait changé. $\mathrm{L}_{\mathrm{a}}$ baisse, pratiquement certaine, de la teneur en matières azotées de la luzerne peut être responsable de cette diminution.

\section{$3^{\circ}$ Evolution au cours des différents cycles de croissance de la luzerne.}

Durant la période de végétation de I964, nous avons suivi les taux d'ammoniac des contenus de deux vaches fistulisées recevant, à l'auge, de la luzerne verte (graph. 3). Pour chaque cycle, nous avons comparé les courbes d'évolution des taux d'ammoniac avec celles des quantités de luzerne consommées (nous n'avons reporté ces consommations que la veille et le jour des prélèvements). Pendant les deux premiers cycles de développement de la luzerne, on a une excellente concordance entre les taux d'ammoniac observés sur les deux vaches et un parallélisme souvent 

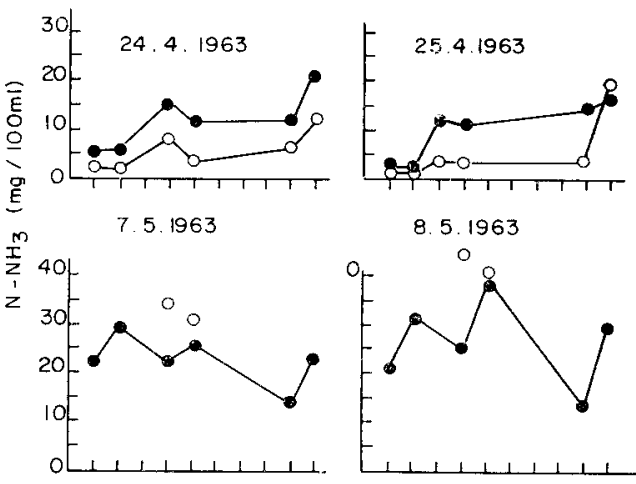

8. 5.1963
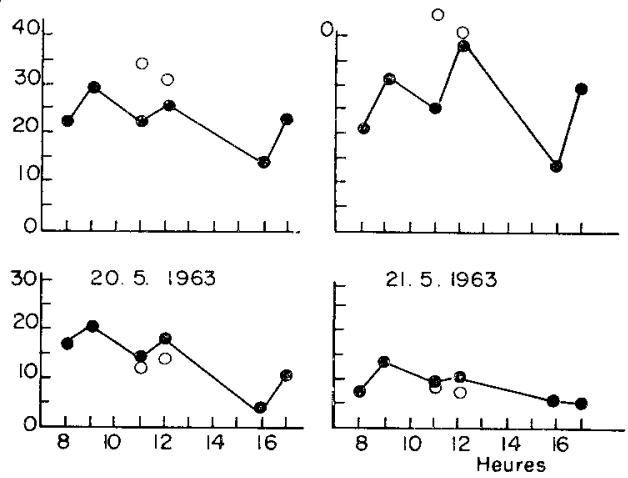

Heures de repas:

\begin{tabular}{|c|c|}
\hline $\begin{array}{l}\text { Concentré . . . } \\
\text { Ensilage } \ldots \ldots \\
\text { Betterave. . . . } \\
\text { Foin . . . . . } \\
\text { Herbe . . . . }\end{array}$ & $\begin{array}{l}C \\
E \\
B \\
F \\
H\end{array}$ \\
\hline
\end{tabular}

Quantités consommées (en $\mathrm{kg}$ de M.S.)

\begin{tabular}{|c|c|c|c|c|}
\hline C & $E$ & B & $F$ & $\mathrm{H}$ \\
\hline 24 avril & $\begin{array}{l}4,4 \\
7,5\end{array}$ & $\begin{array}{l}2,4 \\
1,7\end{array}$ & $\begin{array}{l}3,2 \\
3,2\end{array}$ & $\begin{array}{l}4,2 \\
3,6\end{array}$ \\
\hline 25 avril & $\begin{array}{l}4,4 \\
7,1\end{array}$ & $\begin{array}{l}2,6 \\
2,3\end{array}$ & $\begin{array}{l}3,0 \\
3,0\end{array}$ & $\begin{array}{l}2,8 \\
2,9\end{array}$ \\
\hline 6 mai 0 & & $\begin{array}{l}0,9 \\
0,9\end{array}$ & $\begin{array}{l}3,1 \\
0,8\end{array}$ & $\begin{array}{r}8,9 \\
10,3\end{array}$ \\
\hline $7 \mathrm{mai} O$ & & & & $\begin{array}{l}12,1 \\
14,2\end{array}$ \\
\hline $8 \mathrm{mai}$ & & & & $\begin{array}{l}14,9 \\
16,6\end{array}$ \\
\hline 20 mai & & & & $\begin{array}{l}14,6 \\
13,0\end{array}$ \\
\hline $21 \mathrm{mai}$ & & & & $\begin{array}{l}13,2 \\
13,4\end{array}$ \\
\hline
\end{tabular}

Graphique $\mathrm{x}$. - Evolution des taux d'ammoniac des contenus de rumen de deux vaches fistulisées, au cours de la mise à l'herbe 
très net entre les courbes figurant l'ammoniac et celles de la consommation (exprimée en kilos de matière sèche). Au troisième et an quatrième cycle, les consommations sont plus irrégulières et les taux d'ammoniac sont quelquefois assez différents pour
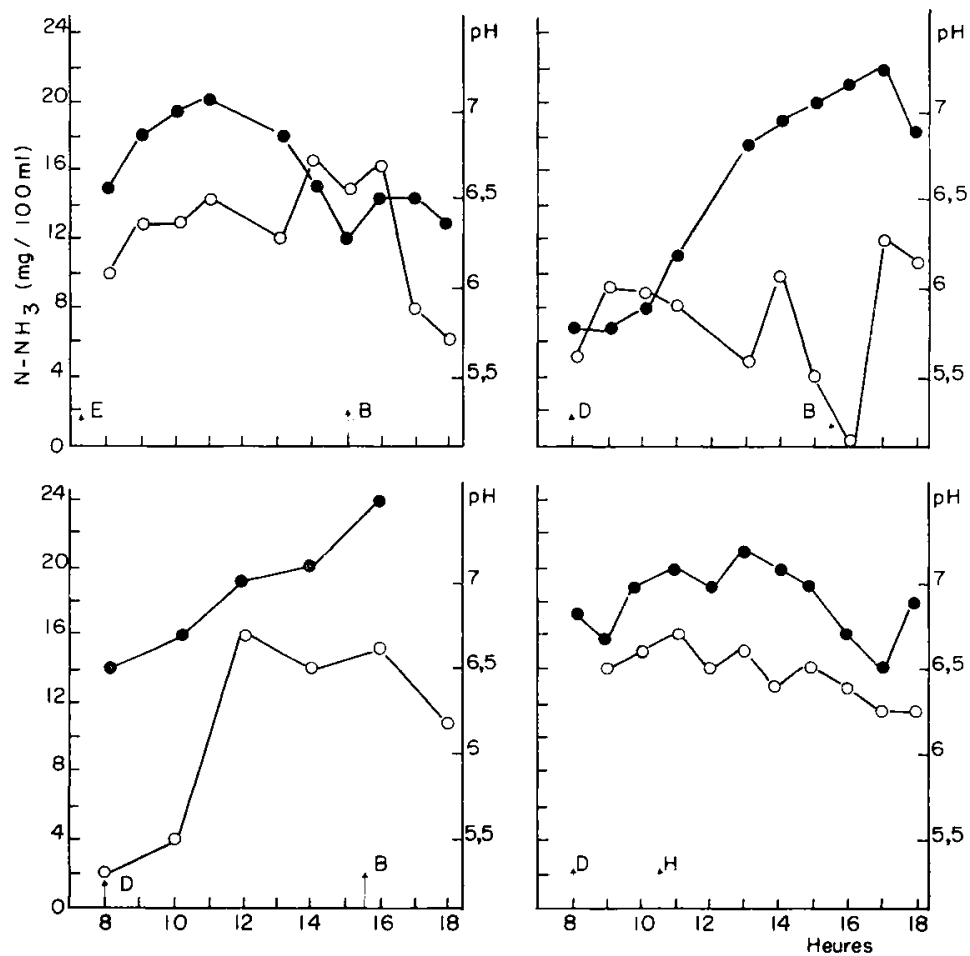

Garphique 2. - Variations de l'ammoniac et du pH du jus de rumen d'une vache fistulisée

- Taux d'ammoniac. soumise a un régime mixte

$\circ \mathrm{pH}$

$\uparrow$ Heures des repas : $\mathrm{E}$ : ensilage; D : drèches ; $\mathrm{B}$ : betteraves ; $\mathrm{IH}$ : herbes.

les deux vaches. Du début à la fin de chaque cycle, les taux d'ammoniac diminuent régulièrement, parallèlement à la teneur en matières azotées de la luzerne consommée. Dans aucun cas, au cours des quatre cycles, on ne dépasse $55 \mathrm{mg}$ d'H $\mathrm{NH}_{3}$ par Ioo $\mathrm{ml}$ de jus.

I,e tableau suivant donne les coefficients d'utilisation digestive apparents de la matière organique et des matières azotées de la luzerne (déterminés sur moutons).

\begin{tabular}{|c|c|c|c|c|c|}
\hline & \multicolumn{2}{|c|}{ CUD-MO } & \multicolumn{3}{|c|}{ CUD-MA } \\
\hline & Début de cycle & Fin de cycle & Début & de cycle & Fin de cycle \\
\hline Premier cycle $\ldots \ldots \ldots \ldots$ & 81 & 75 & & 69 & 57 \\
\hline Deuxième cycle...$\ldots \ldots$ & 82 & 79 & & 66 & 58 \\
\hline Troisième cycle...$\ldots \ldots$ & 81 & 76 & & 69 & 60 \\
\hline
\end{tabular}



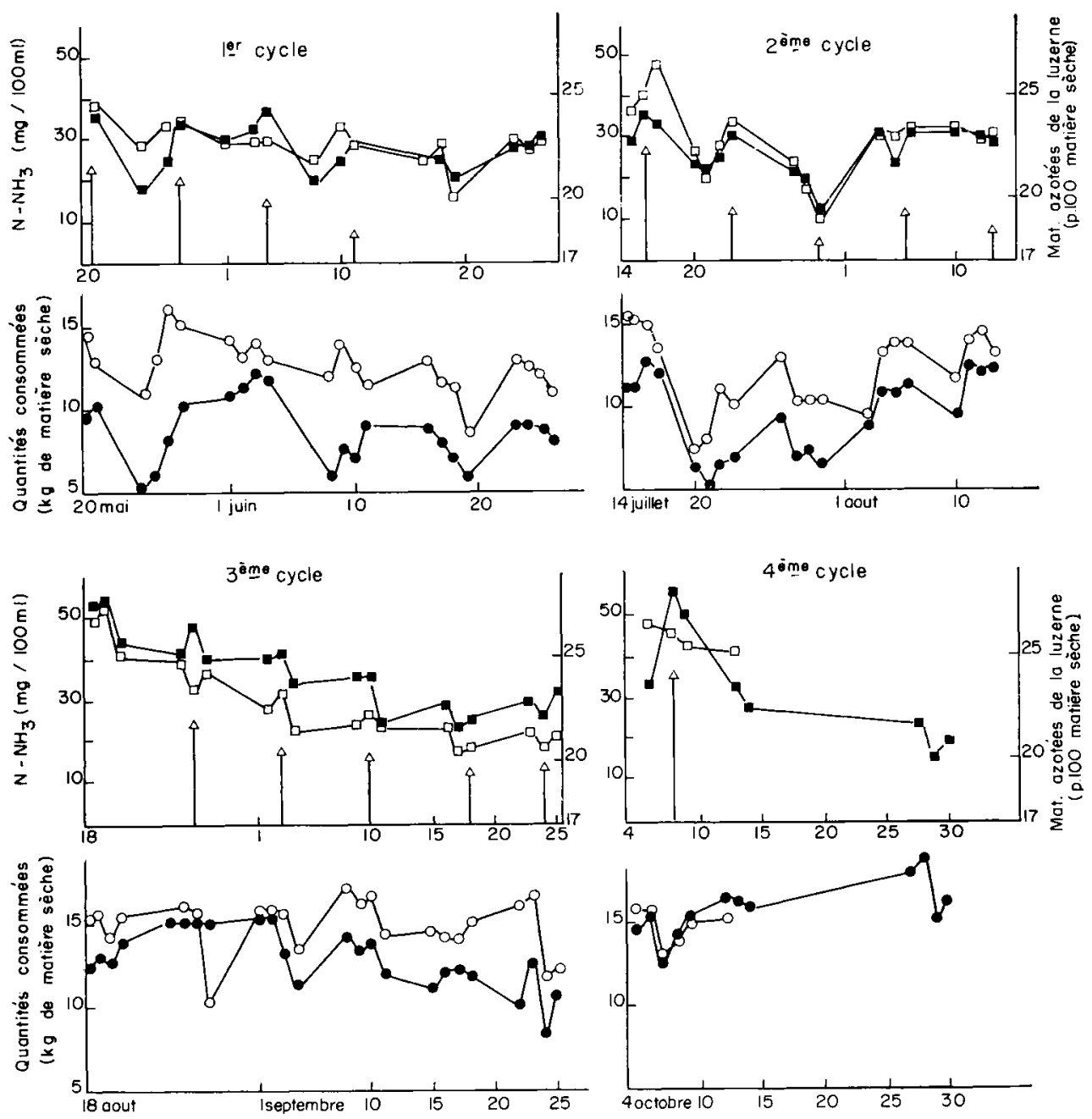

GraPHIQUe 3. -. Varialions des teneurs en ammoniac des jus de rumen et des consommations de deux vaches fistulisées recevant de la luzerne frâ̂che récoltée pendant qualre cycles successifs de végétation.

Taux d'NH Quantités consommées
: vache $\mathrm{n}^{\circ} 778$

: $\square$ vache $\mathrm{n}^{\circ} 77^{8}$
- vache $\mathrm{n}^{0}$ 9027.

o vache $n^{\circ} 9027$. 
Nous avons calculé, pour certains prélèvements, les quantités totales d'ammoniac présentes, deux heures après un repas, dans les rumens de ces deux vaches (tabl. 4), pour ceci, les rumens ont été vidés en totalité et les teneurs en matières sèches calculées. Au premier cycle de croissance de la luzerne, les quantités sont très voisines pour les deux animaux et peu variables $\left(28 \mathrm{~g}\right.$ d' $\mathrm{N} \mathrm{NH}_{3}$ en moyenne pour la vache $778,27 \mathrm{~g}$ en moyenne pour la vache 9027). Au deuxième cycle, elles

\section{TABLEAU 4}

Quantités totales d'ammoniac existant dans le rumen de deux vaches fistulisées recevant de la luzerne verte

Même expérience qu'au graphique $2\left(\mathrm{en} \mathrm{g} \mathrm{d}\right.$ ' $\mathrm{N} \mathrm{NH}_{3}$ )

\begin{tabular}{|c|c|c|c|}
\hline $\begin{array}{c}\text { Cycle de croissance } \\
\text { de la luzerne }\end{array}$ & Date & Vache 778 & Vache 9027 \\
\hline Premier & $\begin{aligned} & 21 . \text { mai } \\
& 1 \text { juin } \\
& 1 \text { juin } \\
& 21 \text { juin } \\
& 26 \text { juin } \\
& \text { Moyenne........ }\end{aligned}$ & $\begin{array}{l}31 \\
\mathbf{2} 6 \\
32 \\
25 \\
27 \\
\frac{28}{28}\end{array}$ & $\begin{array}{l}31 \\
25 \\
26 \\
26 \\
25 \\
27\end{array}$ \\
\hline Deuxième & $\begin{array}{c}17 \text { juillet } \\
20 \text { juillet } \\
10 \text { âout } \\
13 \text { âout } \\
\text { Moyenne .......... }\end{array}$ & $\begin{array}{l}29 \\
20 \\
26 \\
25 \\
20\end{array}$ & $\begin{array}{l}42 \\
24 \\
28 \\
26 \\
24\end{array}$ \\
\hline Troisième & $\begin{aligned} 20 & \text { âout } \\
25 & \text { septembre } \\
\text { Moyenne } & \ldots \ldots \ldots\end{aligned}$ & $\begin{array}{l}46 \\
\frac{28}{37}\end{array}$ & $\begin{array}{l}45 \\
20 \\
32\end{array}$ \\
\hline Quatrième & $\begin{array}{l}9 \text { septembre } \\
30 \text { octobre }\end{array}$ & $\begin{array}{l}43 \\
16\end{array}$ & \\
\hline
\end{tabular}

sont d'abord élevées ( $29 \mathrm{~g}$ pour la vache 778 et $42 \mathrm{~g}$ pour la vache 9027 ) puis diminuent et se stabilisent aux environs de $26 \mathrm{~g}$. L'évolution paraît la même aux troisième et quatrième cycles.

\section{ÉTUDES CINÉTTIQUES}

\section{Expérience III : \\ Étude cinétique sur vache fistulisée}

Dans le but de préciser l'évolution de la production de l'ammoniac au cours de la journée, après des repas de composition variable, nous avons essayé d'effectuer des prélèvements sur une vache fistulisée, âgée et tarie, ayant un mauvais appétit 
(et par conséquent un comportement alimentaire changeant) recevant un régime mixte. Dans ces conditions (cf. graph. 2) nous avons observé une grande variabilité des taux d'ammoniac. Il est très difficile d'interpréter les résultats obtenus sur une journée si l'on ignore comment ont évolué les consommations et le taux d'ammoniac avant la période expérimentale.

\section{Expérience IV}

Prélèvements automatiques et continus sur moutons fistulisés

Le graphique 4 montre les courbes obtenues en effectuant les prélèvements, suivant la méthode décrite, toutes les heures ou toutes les deux heures. L'ensemble de ces prélèvements a été fait sur la même brebis consommant le même foin ad libitum (trois distributions par jour) et le même aliment concentré (un repas par jour).

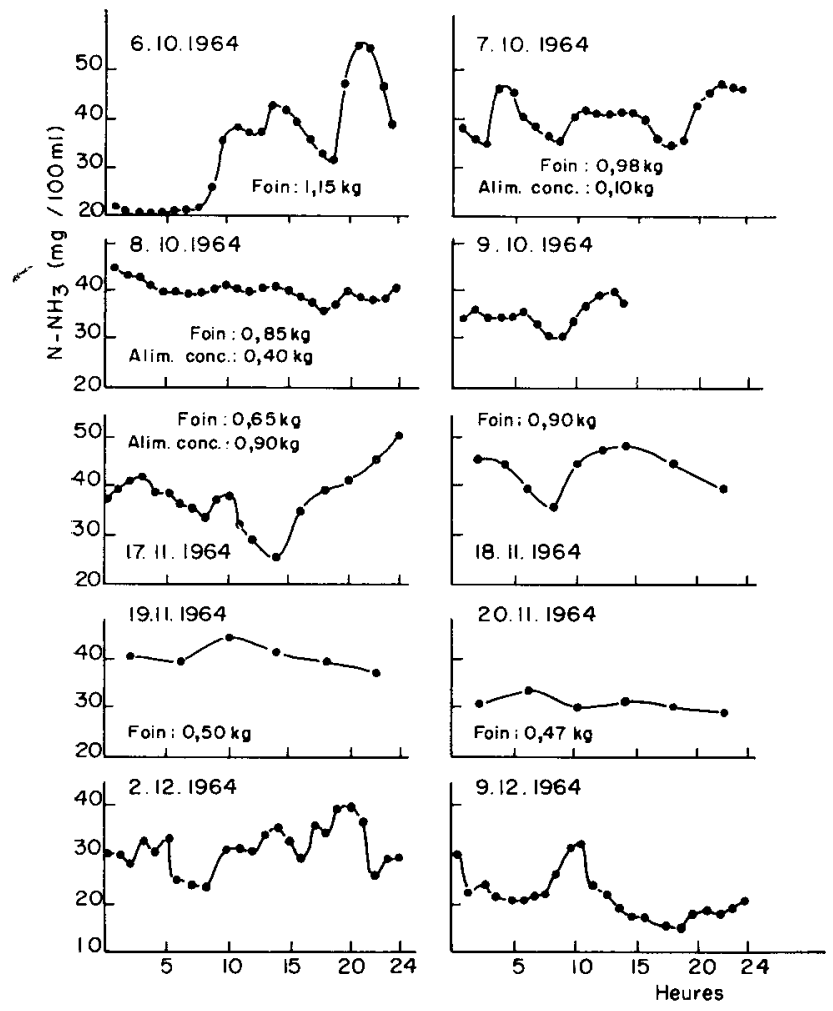

GraphIQUe 4. - Varialions des leneurs en ammoniac des jus de rumen d'une brebis consommant ad libitum un foin et un aliment concentré.

(Trois distributions de foin par jour : 7 h 30 , II $\mathrm{h} 30$, I $7 \mathrm{~h} 30$ ). Foin \% M. S.

Matières azotées totales ...... $\quad \overline{15,3}$

Cellulose brute $\ldots \ldots \ldots \ldots \ldots . \quad 26,6$

Aliment concentré $\quad \%$ M. S.

Matières azotées totales . . . . .

Cellulose brute .......... $\quad 3,7$ 
Le graphique 5 donne deux exemples de périodes de $24 \mathrm{~h}$ pour lesquelles le comportement alimentaire est différent. Dans un cas, il existe des repas entraînant de brusques augmentations de la concentration d'ammoniac; dans l'autre cas, cette

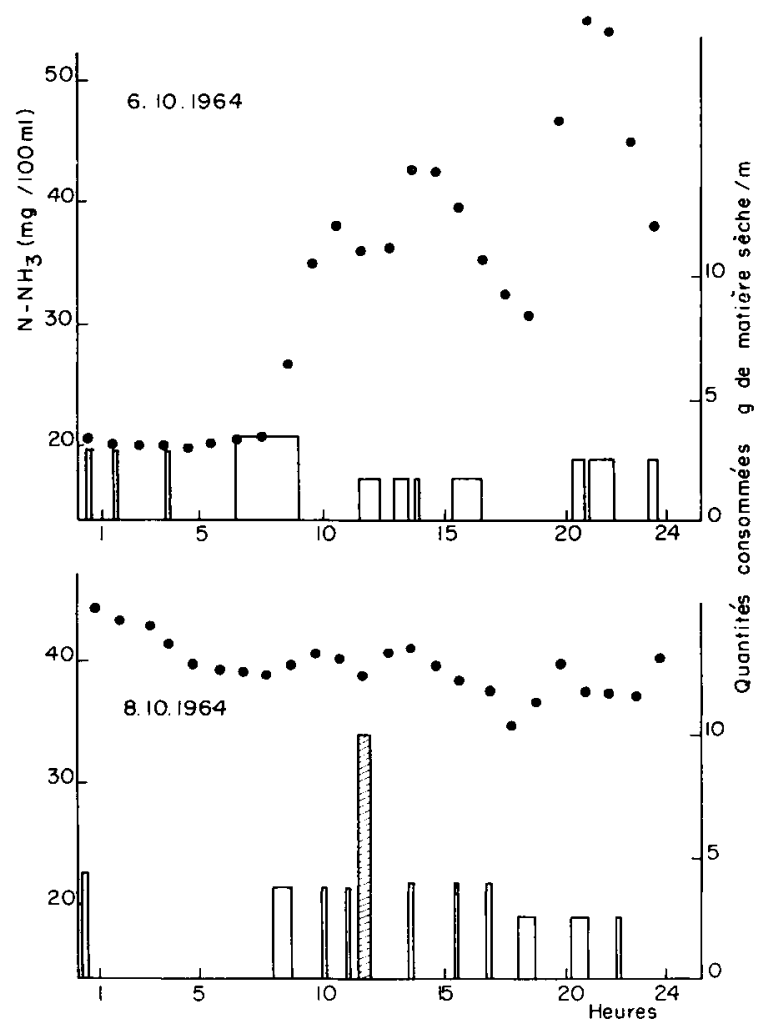

GRAPHIQUE 5. - Variations des leneurs en ammoniac des jits de rumen al comportement alimentaire d'une brebis consommant du foin seul $\square$ ou du foin associé à un aliment concentré $\mathscr{D}$. Prélèvements automatiques et continus.

concentration demeure élevée et peu variable par suite d'une consommation régulièrement répartie dans le temps. Dans l'ensemble des périodes, les concentrations d'ammoniac, à une heure donnée, sont très variables d'un jour à l'autre, bien que le mode d'alimentation demeure rigoureusement le même.

\section{DISCUSSION}

\section{A. - Méthodes}

Les difficultés d'interprétation des quelques résultats que nous venons de présenter expliquent que nous ayons été amenés à revoir nos méthodes. 
B. GAUSSERES

\section{Io Méthode de prélèvement}

Sur animaux abattus.

Les résultats du tableau I provenant d'échantillons prélevés sur des animaux d'abattoirs ne permettent pas de suivre avec précision la transformation de la fraction azotée de la ration au niveau du rumen. Ils ne donnent tout au plus que des ordres de grandeur. L'échantillonnage, dans ces conditions, est difficile et les contenus des différentes parties du tube digestif peuvent se mélanger au cours de l'abattage. On observe alors des différences individuelles assez importantes, surtout lorsque l'abattage est effectué plus de cinq heures après le repas. Si l'abattage a lieu immédiatement après le repas et si les niveaux de consommation des différents animaux sont voisins, les variations individuelles sont plus faibles, le dernier repas constituant une partie plus importante du contenu du rumen (20 p. roo à 50 p. Ioo selon le régime et le nombre de repas).

\section{Sur animaux fistulisés.}

Les prélèvements sur animaux porteurs de canules peuvent être également peu satisfaisants suivant la manière dont on procède. Le contenu du rumen n'a pas les mêmes caractéristiques physiques et chimiques suivant la zone dans laquelle il est prélevé. La partie ventrale est plus liquide (avec la plupart des régimes) et le pH augmente de la partie postérieure à la partie antérieure ; la zone dorsale postérieure est la plus acide; il peut y avoir plus d'une unité de $\mathrm{pH}$ d'écart entre deux zones (observations personnelles sur vaches). I a constitution d'un échantillon moyen représentatif de l'ensemble est difficile si l'on ne vide pas totalement le rumen. On soumet alors la masse en digestion à une aération importante qui ne permet pas d'effectuer plusieurs prélèvements après un même repas, car les orientations fermentaires risquent de changer après la première manipulation.

Nous avons donc pensé qu'il était préférable, dans un premier temps, d'étudier la digestion en suivant l'apparition des produits terninaux solubles, en un point du rumen qui soit toujours le même (prélèvements automatiques). Nous avons choisi comme lieu de prélèvement la partie centrale du sac ventral. I lans cette zone, la phase liquide est susceptible de transiter rapidement vers le feuillet et la dilution par la salive n'est probablement pas importante. Les mouvements de liquide à l'intérieur du rumen sont tels que pendant les cinq minutes (ou dix minutes) que dure le prélèvement (cas des prélèvements automatiques) on a une série de cycles de contractions (un par minute environ sur vache d'après $\mathrm{BALCH}, \mathrm{Ig} 6 \mathrm{I}$ ) et donc à chaque cycle, une remontée du liquide ventral à travers la zone dorsale et ainsi, un renouvellement du liquide ventral. I'échantillon obtenu représente la fraction qui transite vers le feuillet sinon celle contenue dans l'ensemble du rumen ( $\mathrm{pH}$ variables). I1 serait sans doute opportun d'observer les fermentations en plusieurs points fixes du rumen.

Quoi qu'il en soit, les résultats obtenus jusqu'à présent par prélèvements continus, sont encore parfois difficiles à interpréter. I,es courbes présentent souvent des irrégularités et des discontinuités dues sans doute pour une part à la consommation de l'eau (qui n'était jusqu'ici contrôlée que globalement (1) et aussi à ce que

(1) Un dispositif enregristreur nous permet actuellement de suivre en continu la consommation d'eau. 
la vitesse d'ingestion des aliments n'est pas constante (sauf peut-être dans les cas ou l'aliment est très homogène).

Le choix du lieu et de la méthode de prélèvement est un problème complexe, si l'on veut obtenir un échantillon représentatif des orientations fermentaires que l'on cherche à déterminer.

\section{$2^{\circ}$ Méthode d'analyse}

Suivant la méthode utilisée, ce que l'on appelle azote ammoniacal ne correspond pas toujours aux mêmes composés. Une étude récente de HiLL et MaNGan (I964) montre que $3 \mathrm{p}$. Ioo à $\mathrm{x} 6 \mathrm{p}$. Ioo des bases volatiles dosées par distillation étaient de la méthylamine. La méthylamine augmenterait après le repas pour atteindre un maximum après $5 \mathrm{~h}$ environ. MiCHEL (communication personnelle) a trouvé dans le contenu du rumen d'une brebis consommant du foin de luzerne $I 9,2 \gamma$ de méthylamine et $22,8 \gamma$ d'ammoniac par $\mathrm{ml}$ de jus.

\section{$3^{\circ}$ Expression des résultats}

Nous avons choisi d'exprimer les résultats en mg d'azote ammoniacal par roo $\mathrm{ml}$ de jus de rumen puisque ce mode d'expression est le plus habituel etqu'il correspond à la valeur donnée par le dosage.

Dans quelques cas (tabl. 2 et 4), nous avons calculé les quantités totales d'ammoniac présentes dans le tumen. Ce calcul est fait en tenant compte du poids total de contenu et de sa teneur en eau. On obtient ainsi un volume théorique qui ne correspond pas obligatoirement au volume réel de répartition de l'ammoniac. Dans les cas particuliers présentés ici, il ne semble pas préférable (compte tenu de la difficulté de détermination des volumes) de transformer les concentrations en quantités totales. Ce mode de calcul diminue l'hétérogénéité de certaines données mais l'augmente pour d'autres. Certains préconisent de ramener la quantité totale d'ammoniac à la quantité d'azote ingéré, on ne peut alots plus se contenter de la quantité totale instantanée mesurée à un moment donné. Il faut connaître les variations des quantités totales en fonction du temps. On est, cependant, amené à donner alors des valeurs qui cherchent à représenter les " pertes » d'ammoniac consécutives à l'ingestion d'une matière azotée donnée. Or, il est difficile d'admettre de telles valeurs quand on ignore tout de l'origine exacte de cet ammoniac et de son devenir.

\section{B. - DISCUSSION GÉNÉRAI,E}

En effet, quelle que soit la façon dont on veuille exprimer la formation de l'ammoniac, pour interpréter les valeurs trouvées, il est nécessaire de connaitre les facteurs de la digestion et du métabolisme de 1'azote.

L'ammoniac présent dans le rumen est le résultat d'un équilibre entre la production et les différentes voies d'utilisation : métabolisme dans le rumen, absorption et évacuation vers le feuillet. Outre ces variations dynamiques, il existe des variations passives dues à la salive, à la consommation, aux échanges d'eau à travers la paroi du rumen. 


\section{Io Formation de l'ammoniac - Les facteurs de formation}

L'action protéolytique et désaminante des microorganismes du rumen a été montrée par de nombreux auteurs (WARNER, I956 ; EI--SHALZY, I952 ; LEWIS, I955 ; BIACKBURN et HobSON, I962) et notamment par OTAGAKI et al. (r955) avec de la caséine marquée au ${ }^{14} \mathrm{C}$, concluant à la formation d'acides gras et d'ammoniac à partir des protéines. On a longtemps considéré les bactéries comme les principales responsables de cette désamination, mais depuis quelques années ò̀ l'on accorde plus d'intérêt aux protozoaires qui peuvent parfois se trouver en quantités considérables (plus de ro g secs par litre de jus, FAUCONnEAU et GaUSSEREs, I96I), on a constaté (KANDATSU et TAGAHASHI, I962; AKKADA et HowARD, I962) qu'ils pouvaient hydrolyser les protéines puis désaminer les acides aminés. Chalmers et al. (I954), LEWIS et MC DONALD (I958) ont effectué des études assez complètes, mais malheureusement avec des animaux qui étaient maintenus dans des conditions expérimentales asse $z$ artificielles, puisqu'ils étaient entraînés à consommer leur ration journalière en une heure. Il est certain que dans de telles conditions, partant d'un niveau de nutrition de la flore assez bas (et donc d'une population sans doute altérée) on amène brusquement une très grande quantité d'éléments nutritifs (par la fraction soluble surtout), ce qui oriente les fermentations dans un sens particulier et peut entraîner l'accumulation momentanée de certains produits terminaux et intermédiaires de la digestion. D'autres auteurs tels que Johns (I956) ou Hogan (I965) se sont intéressés à la digestion au pâturage et notamment aux premiers jours qui suivent la mise à l'herbe. Les fermentations qui ont lieu pendant cette période sont aussi probablement assez spéciales. La flore doit s'adapter à un substrat nouveau. Cependant, cette adaptation n'est peut-être pas le seul phénomène qui entre en jeu.

Des résultats exposés précédemment (graph. I), on pourrait déduire qu'au moment de la mise à l'herbe, la production d'ammoniac est intense, puis qu'après une période d'enviton $\mathrm{I}_{5}$ jours (dans ce cas particulier au moins) permettant une adaptation de la population microbienne (soit par augmentation de l'utilisation, soit par diminution de la production) on retrouve un taux d'ammoniac assez faible.

Mais une diminution du taux d'ammoniac peut aussi être due à une diminution de la teneur en matières azotées de l'herbe, d'où une consommation moins importante de matières azotées. Dans nos résultats (graph. 3) on constate qu'il y a une relation étroite entre le niveau de consommation et le taux d'ammoniac. Hogan (Ig63) pense également que pour un aliment donné il y a une relation entre le niveau de consommation et le taux d'anmoniac. Le niveau de consommation, ou plus précisément la consommation au cours des 24 h ou des $48 \mathrm{~h}$ précédant le dosage, peut agir en modifiant la quantité d'urée endogène arrivant au niveau du rumen : par la salive (SOMERS, Ig6I) ou directement à travers la paroi (ENGEIHARDT, I963). L'apport endogène peut ainsi n'être pas négligeable dans certaines conditions d'alimentation. ENGELHARDT (I963), travaillant sur chèvres, estime qu'il y a un passage d'urée, du sang vers le rumen, variant entre $\mathrm{I}, 8$ et $\mathrm{I} 7,8 \mathrm{~g}$ par $24 \mathrm{~h}$ si l'on admet un taux moyen d'urée sanguine de $25 \mathrm{mg}$ par Ioo $\mathrm{ml}$ de sang. MoIR et HARRIs (I962), estiment à $3 \mathrm{~g}$ d'azote l'entrée d'urée dans le rumen d'agneaux. Sur moutons, Somers (I96I) trouve que pour une ingestion de $14,8 \mathrm{~g}$ d'azote par jour, près de $300 \mathrm{mg}$ sont recyclés dans le rumen sous forme d'urée salivaire (ce qui ne représente que $2,2 \mathrm{p}$. IOO). DECKER 
et al. (I960) ont montré que l'urée synthétisée dans le foie passait dans le rumen. GAERTNER et al. (I96I) ont cependant montré que le transfert actif du sang vers le rumen était indépendant de la concentration sanguine.

A composition azotée équivalente, le mode de présentation semble jouer un rôle important (nos résultats, tabl. 3). La structure physique peut déterminer une certaine " disponibilité " et intervenir sur la vitesse, l'intensité de la digestion et le temps de séjour dans le rumen et, par là, sur les quantités respectives des différents produits terminaux. Le même phénomène s'observe quand les matières azotées ont subi des traitements technologiques tels que chauffage ou imprégnation par les tannins (IEROY et al., I964).

Après un repas, habituellement, l'ammoniac augmente atteignant un maximum après I h à $4 \mathrm{~h}$, puis revient à un niveau comparable à celui que l'on avait avant le repas. Pour un animal donné ayant un régime donné, certains auteurs observent une reproductibilité assez bonne dans ces courbes (ChALMERs et SYNGE, r954). Pour ceci, il faut que 1'animal effectue son unique repas journalier rapidement, le niveau d'alimentation demeurant le même. Dans nos résultats (tabl. I et graph. 4), pour un régime donné, on retrouve souvent avant le repas des taux d'ammoniac très différents, dans des conditions d'alimentation normale. De plus, si la quantité ingérée pendant le repas varie (donc la quantité de protéines ingérées) la production d'ammoniac est plus ou moins importante et s'étale sur un temps plus ou moins long dépendant de la durée de la consommation. Enfin, quelquefois, sans qu'il y ait une nouvelle consommation, on observe $5 \mathrm{~h}$ à $8 \mathrm{~h}$ environ après le repas, une nouvelle augmentation du taux d'ammoniac. BLACKBURN et Hobson (I962) ont constaté le même phénomène sans pouvoir l'interpréter. Cette nouvelle augmentation peut être due soit à une autolyse microbienne amenant une nouvelle source d'azote, soit à une protéolyse plus intense suivie de désamination (après la phase de multiplication des micro-organismes), soit d'un retour d'urée par les voies sanguines, soit encore à une production de méthylamine (HII, I, et MANGAN, I 964 ) dont le maximum de concentration se situe entre 3 et $5 \mathrm{~h}$ après le repas.

Si le comportement alimentaire ne change pas, si le niveau de consommation demeure le même, on obtient après les repas (même quantité consommée, même temps de consommation) des cinétiques de production d'ammoniac qui sont semblables Ceci, ne demeure cependant vrai que si l'on considère comme constants les autres facteurs liés aux aliments ou à la physiologie de l'animal. L'influence, par exemple, des glucides de la ration sur les concentrations d'ammoniac du rumen a fait l'objet de nombreuses études discutées en détail par LEWIS (I g60). En somme, si l'on tient les animaux dans des conditions expérimentales rigoureusement stables (repas en une heure par exemple), on a toutes chances d'observer un processus digestif qui sera reproductible, mais qui n'aura pas pour autant une signification nutritionnelle générale, du moins, si ces conditions sont trop artificielles.

\section{$2^{\circ}$ Utilisation de l'ammoniac}

Les micro-organismes, générateurs d'ammoniac, en sont également consommateurs. Gilroy (I957) a montré sur des cultures pures de bactéries isolées de contenus de rumen que 50 p. Ioo d'entre elles utilisaient seulement l'ammoniac pour synthétiser des protéines, les autres ayant besoin d'acides aminés pour se développer. 
BRYAN'T et RoBINSON (I962) ont trouvé que sur 89 souches isolées du rumen, 1'ammoniac est indispensable pour $25 \mathrm{p}$. Ioo d'entre elles et que pour $56 \mathrm{p}$. Ioo (y compris les 25 p. Ioo précédents) on a un développement satisfaisant en présence d'ammoniac seulement.

- S'il n'est pas métabolisé par les micro-organismes, l'ammoniac est susceptible d'être absorbé. Si l'étude qualitative de cette absorption est relativement simple, l'évaluation quantitative en est délicate par suite des difficultés d'appréciation du débit sanguin. LEWIS (I 957) a travaillé dans des conditions telles que le taux d'ammoniac augmentait beaucoup pendant une période brève, ce qui justifie une absorption èlevée. D'aprés HoGax (Ig6I), il y aurait diffusion vers le sang sous forme $\mathrm{NH}_{3}$. Yoshida (I963) estime que l'absorption de la forme non ionisée est plus intense. Il semble que l'on puisse admettre que la concentration, la quantité d'acides gras volatils et le $\mathrm{pH}$ sont les principaux facteurs qui règlent 1'absorption. LEwIs (I957) évalue cette absorption à I $4 \mathrm{~g}$ d'azote ammoniacal par $24 \mathrm{~h}$, pour des moutons recevant $800 \mathrm{~g}$ de foin et $50 \mathrm{~g}$ de caséine. Ce chiffre est très élevé (conditions expérimentales).

- Les transports de l'ammoniac et de l'urée sont essentiellement fonction de leurs concentrations dans le sang et dans le rumen. Si les orientations fermentaires dans le rumen dépendent avant tout des aliments, les concentrations sanguines sont le reflet du métabolisme général de l'animal. Cet aspect du problème est assez mal connu, mais quelques études font penser que l'on peut difficilement appliquer au ruminant les concepts classiques admis chez le monogastrique : READ (I952), HouPT (I959), MC LaRen et al. (ig62), Juha'sz (Ig62), Wa'Tson et al. (I949), SchMidT-NielSEN et OSAKI (I958). Ces auteurs ont observé des phénomènes originaux concernant l'utilisation métabolique de l'urée. On sait, en outre, que chez le monogastrique, l'urée peut être assinilée (après transformation par l'uréase microbienne du tube digestif). LARDY et FEIDOTT (1949) et RosF et al. (I949) ont montré que les sels d'ammonium pouvaient être utilisés dans la synthèse des acides aminés non essentiels. De plus, on a observé que le ruminant "s'adaptait " à une alimentation riche en urée (SMr'sH et al., I960; MC LAREx, I964), cette adaptation n'étant peut être pas due uniquement à la modification du faciès microbien.

Enfin, les besoins azotés d'entretien déterminés récemment par EiLLIOTT et Topps (I963) sont sensiblement le tiers de ceux préconisés par BRODY, ce qui semble indiquer que le type de la ration et son mode de présentation interviennent au moins autant que sa composition chimique, en particulier, pour les faibles niveaux d'alimentation.

Rę̧u pour publication en décembre 1965.

\section{SUMMARY}

AMMONIA IN THE DIGESTIVE TRACT OF RUMINANTS : ITS SIGNIFICANCE

The samples of rumen contents analysed were taken by three methods. They were from : Slaughtered cattle (tables $I$ and 2 ).

From cattle with fistulæ by withdrawal from different parts of the rumen; the cannula remained open (table 3 and 4 ; graphs I, 2 and 3 ). 
From a ewe with a fistual by means of a filter valve attached to the cannula which remained in place. The pump operated automatically at regular intervals of time (graphs 4 and 5 ).

Altogether 156 animals and 17 diets were studied. Very high values comparable to some quoted in the literature (100 $\mathrm{mg} \mathrm{NH}_{3}-\mathrm{N}$ per $100 \mathrm{ml}$ ) were never found. On the other hand there were very large differences between individual animals in the same physiological state and on the same diet.

In two cows with fistulæ given the same hay long or ground and pelleted (table 3 ) the amount of ammonia in relation to the intake of feed was less when the hay was pelleted.

For two cows with fistulæ the pattern of changes in ammonia content was studied when they went out to pasture (graph I).

In two cows with fistulæ given fresh luceme in mangers during four vegetative cycles the concentration of ammonia varied in the same direction as the amount of lucerne eaten and as the crude protein content of the lucerne.

The different results are discussed in relation to the method of sampling and to the nutritional significance of the ammonia estimated.

\section{RÉFÉRENCES BIBLIOGRAPHIQUES}

Akkada A. R. A., Howakd B. II., Ig62. 'The biochemistry of rumen protozoa. Bioch. J., 82, 3 г3.

Annison F. F., Chalmers M. I., Marshall S. B. H., Sxnge R. L. M., i954. Ruminal ammonia formation in relation to the protein requirement of Sheep. J. agric. Sci., 44, 270-273.

BALCII C. C. Movement of digesta through the digestive tract. In Digestive physiologie und nutrition of the ruminant (L.EWIs) white friars Press. Londres, 23-33.

Blackburn T. H., Ilobson P. N., ig62. The degradation of protein in the rumen of the sheep and redistribution of the protein nitrogen after feeding. Rowett Research Institute. Ann. Rep. Anim. Nutr., 18, I2.

BRYANT M. P., Robinson I. M., 1962. Some nutritionnal characteristics of predominant culturable ruminal bacteria. J. Bactériol., 84, 605.

Cilalmkrs M. I., Synge R. L. H., I954. Ruminal ammonia formation in relation to the protein requirement of sheep. J. agric. Sci., 44, 263-269.

Decker P., Gaertner K., Hili, H., Holler Il., Ifornicke H., 1960. Metabolism of C14 urea in lactating goats. Fifth intemat. Congr. nulr. Abstr., $11^{\circ} 39$.

Mc Donalij I. W., 1948. Studies in the metabolism of the sheep with special reference to the digestion of protein. Disserlation Univ. Cambridge.

MC Donald I. W., I952. The role of ammonia in ruminal digestion of protein. Bioch. J., 5\%, 86-96.

MC DonALI) I. W., 1954. The extant of conversion of food protein to microbial protein in the rumen of the sheep. Bioch. J., 66, i 20 .

Mc Donali I. W., Haj,L R. J., 1957. The conversion cf cascin into microbial protein in the rumen. Bioch. J., 6r, 400 .

Elliotr R. C., 'Tops's J. H., 1963. Studies of protein requirement of ruminants. Brit. J. Nutr., 1, 539-547.

El-Shatzy K., 1952. Degradation of protein in the rumen of the sheep. Bioch. J., 51, 647.

ENGELIIARD! W., I963. Water exchange in the rumen wall. Naturwissenschaften., 50, 357-358.

Fauconneau G., Gausseres 13., i96r. Les bactéries et les protozoaires libres du rumen; variations avec le régime alimentaire et le repas. Congrès de Hambourg, 32-34.

Fauconneat G., Gausseres 13., 196r. Les bactéries et les protozoaires libres du rumen; variations avec le régime alimentaire et le repas - production d'ammoniac. Ann. Nutr. Alim., 15, $291-299$.

Gaertnf:R K., Decker P', IIILl H., I96I. Passage of urea and ammonia across the rumen wall of the gcat. Arch. Ges. Physiol., $274-281$.

GARTNER K., I963. Regulation of the ammonia concentration in the rumen and effect on the antidiurectic hormone content of serum after sudden changes of intraruminal ammonia. Deutsch. Tierärztl. Wochenscht., $70,16-20$.

Gausseres B., Fauconnesu (i., I g65. Évaluation quantitative à l'atide de sa teneur ell acides nucléiques de la population microbienne du tube digestif des ruminants. I. Méthode et application aux contenus de rumen de bovins recevant différents régimes. Ann. Biol. anim. Bioch. Biophys., 5, 5-2o.

Gausseres 13., I965. Méthode de prélèvement automatique de la phase liquide des contenus de rumen. Ann. Biol. anim. Bioch. Biophys. 5, 407-4II.

Gilroy J. J., I957. Nitrogen requivements for the grozith of bovine rumen bacteria. Ph. D. Thesis University of Maryland, College Park, Md.

HILL K. J., MaNGaN J. L., I 964 . The formation and distribution of methylamine in the ruminant digestive tract. Bioch. J., $93,39-45$. 
Hogan J. P., ig6r. The absorption of ammonia through the rumen of the sheep. Austral. J. Biol. Sci., $14,448-460$.

Hogan J. 1', I963. Digestion of food by the grazing sheep. Austral. J. agric. Res., 15, 397-407.

Hogax J. P., I964. The digestion of food by the grazing sheep. Austral. J. agric. Res., 15, $397-407$.

Houpt T. R., 1959. Utilization of blood urea in ruminants. Ann. J. Physiol, 197, I 5.

Jorns A. T., 1956. Pasture quality and ruminant digestion. New. Zeal. J. Sci. Technol., 37, 234-239.

JuHA'sz B., I962. Effect of starvation on ammonia content and $\mathrm{pH}$ of the rumen fluid and on urea, cholesterol and sugar in the blood. Acta Vet. Hung., 12, 383-395.

Kandatsu M., Takainshi. N studies on reticulo-rumen digestion. XXIII. Nitrogen metabolism of infusoria. Jap. J. Zootech. Sci., 34, 148-155.

LARDY M. A., FELbotT G., 1949. The net utilization of ammonia nitrogen by the growing rat. J. Biol. Chem., 179, 509 .

Mc Itaren G. A., Anuerson G. C., Barth K. M., Welch J. A., ig62. Casein and its degradation products in the utilization of urea nitrogen by lambs. J. anim. Sci., 21, $25^{8}$.

Mc LAREN 6. A., 1964. Symposium on microbial digestion in ruminants; nitrogen metabolism in the rumen. J. anim. Sci., 23, 577-590.

Leroy F., Zelter S. Z., Françols., A C. Protection des protéines alimentaires contre la désamination. bactérienne au niveau du rumen. F́tudes en rumen artificiel. C. R. Acad. Sci., Paris, 259, I 592 -I 595 .

LEWIS D., 1955. Amino acid metabolism in the rumen of the sheep. Brit. J. Nutr., 9, 215.

LEWIS D., 1957. Blood-urea concentration in relation to protein utilization in the ruminant. J. agric. Sci., 48, $43^{8}$.

Lewis D., MC Donali) 1. W., I958. The inter-relationships of individual proteins and carbohydrates during fermentation in the rumen of the sheep. J. agric. Sci., 5\%, 108.

LEwis D., 196r. The inter-relationships of individual proteins and carbohydrates during fermentation in the rumen of the sheep. J. agric. Sci., 58, 73-79.

LEWIS D., I961. Digestive physiology and nulrition of the mininant. Butterworth's, Londres, Angleterre.

LEWIs D., 1962. The inter-relationships of individual proteins and carbohydrates during fermentation in the rumen of the sheep. J. agric. Sci., 58, 73-79.

Littie C. O., Burrousu S. W., Woovs W., r963. Nutritional significance of soluble nitrogen in dietary proteins for ruminants. J. anim. Sci., 22, $35^{8-36}$.

Moir R. J., Harris I. E., I962. Ruminal fora studies in the sheep. X. Influence of nitrogen intake upon ruminal formation. J. Nutr., 77, 285.

OTACAKI K. K., BLACK A. L., Goss H., KLEIBER H., I955. In vitro studies with rumen micro-orga nisms using carbon-I 4 labeled casein, glutamic acid, leucine and carbonate. J. agric. Food. Chem., 3, 948.

Rose W. C., SutTI L. C., Wounck M., Shane M., 1949. The utilization of ammonium salts, urea and other compounds in the synthesis of non essential amino acids in vivo. J. Biol. Chem., 181, 307.

RLCkBusi Y., I963. Kecherches sur la régulation centrale du comportement alimentaire ches les ruminants. Theses, Univ., Lyon, 215 .

Schindi-Nielsen B., OSAKi H., 1958. Renal responses to changes in nitrogen metabolism in sheep. Ann. J. Physiol., 193, 657 .

Smitil G. S., Dunbar R. S., Mc Lares G. A., Anderson G. C., Welch J. A., ig6o. Measurement. of the adaptation response to urea-nitrogen utilization in the ruminant. J. Nutr., 71, 20.

Somers Mi., rg6r. Factors influencing the secretion of nitrogen in sheep saliva. Austral. J. Exper. Biol. $39,145^{-} 56$.

SutTon J. D., Mc Gilimard, Jacobson N. L., 1963. Functional development of rumen mucosa. I. Absorptive ability., 4, 426-436.

Underwood E. J., Morr R. J., I956. Protein utilization in the ruminant. Proc. 7th Internat. Grassland Congr., $241-245$.

Warner A. C., 1956. Protcolysis of rumen nicro-organisms. J. Gen. Microbiol., 14, 749.

Watson C. J., Davinson W. M., Kennlidy J. W., 1949. The nutritive value of nitrogenous compounds for ruminants. Sci, Agric, 29, $185-188$.

Weller R. A., Gray F. V., PILGrim A. I'., I95\%. The conversion of plant nitrogen to microbial nitrogen in the rumen of the sheep. Brit. J. Nutr., 12, $42 \mathrm{I}-429$.

Yoshina J., Nakamura R., Ig6.3. Studies on the toxicity of urea and its control. Jap. J. Zootech. Sci., 34, 29-31. 